\title{
OTIMIZAÇÃO DO PROCESSO DE EXTRAÇÃO DO ÓLEO DE SEMENTE DE MORINGA POR ULTRASSOM
}

\author{
N. W. SIQUEIRA ${ }^{1}$, F. N. C. ALMEIDA ${ }^{1}$, S. H. B FARIA ${ }^{1}$ e N. C. PEREIRA ${ }^{1}$ \\ ${ }^{1}$ Universidade Estadual de Maringá, Departamento de Engenharia Química \\ E-mail para contato: nwsiqueira@gmail.com
}

\begin{abstract}
RESUMO - Este trabalho objetiva determinar as melhores condições para extração assistida por ultrassom do óleo de semente de moringa, utilizando a metodologia de superfície de resposta. Para isso, foi utilizado o hexano como solvente e realizado um delineamento fatorial composto central rotacional (DCCR). As variáveis independentes analisadas foram o tempo de extração $(30,60$ e $90 \mathrm{~min})$, temperatura do banho ultrassônico $\left(25,35\right.$ e $\left.45^{\circ} \mathrm{C}\right)$ e a razão de mistura (1:6, 1:8 e 1:10 amostra/solvente), sendo o teor de óleo a variável resposta. A melhor condição de extração do óleo foi obtida em menor temperatura, maior tempo e razão de mistura. O teor variou entre $25,75 \%$ e $39,80 \%$, de forma que, o maior valor foi encontrado em 60 minutos, $25^{\circ} \mathrm{C}$ e razão de mistura $1: 10$.
\end{abstract}

\section{INTRODUÇÃO}

Moringa oleifera Lamarck é uma planta que pertence à família das Moringaceae, nativa da Índia e amplamente cultivada nos trópicos de todo o mundo. Sua semente é composta em grande parte por óleo e proteína, tal que os seus teores podem variar de acordo com a região do plantio em função das condições climáticas e geológicas do meio (Anwar e Bangher, 2003). Segundo Dahot (1998), a semente apresenta 38\% do seu peso composto por óleo que é constituído de glicerídeos dos ácidos oléicos $(63,4 \%)$, palmítico $(8,3 \%)$, esteárico $(8,0 \%)$ e linoléico $(3,1 \%)$.

O óleo extraído da semente apresenta alto valor alimentício e industrial. É claro, doce, inodoro e resistente a rancificação, apresenta alta qualidade e pode ser usado no preparo de alimentos, na fabricação de sabonetes, cosméticos e combustíveis (Dahot, 1988). O elevado percentual de ácido oleico favorece a obtenção de biodiesel a partir deste óleo, devido ao baixo teor de insaturações, que tem reflexo direto e positivo na sua estabilidade à oxidação, facilitando o transporte e armazenamento (Santana et al., 2010).

Os métodos convencionais para extração de óleos de sementes oleaginosas são a prensagem e a extração por solventes. A prensagem mecânica fornece óleo de alta qualidade, no entanto em grande parte dos casos, o processo de extração proporciona um baixo teor e consome grande quantidade de energia. Já a extração por solventes possibilita a remoção de praticamente todo o óleo extraível, porém é necessária uma grande quantidade de solvente e tempo (Tian et al., 2013).

O ultrassom é um processo de extração não convencional, que utiliza a energia de ondas sonoras geradas em frequência superior à capacidade auditiva do ser humano. Estas ondas 
sonoras criam uma variação na pressão do líquido empregado no processo, gerando cavitação (Melecchi, 2005). O ultrassom tem sido reconhecido por aplicação potencial na extração de óleos, proteínas e compostos bioativos de plantas ou animais (Goula, 2013).

A eficiência desta técnica de extração é citada como sendo igual ou melhor do que a obtida com o extrator Soxhlet, apresentando as vantagens de alta reprodutibilidade, possibilidade de utilização para uma ampla faixa de tamanhos de amostra, rapidez de processamento e baixo custo (Sargenti e Vichnewsi, 2000).

Sabendo que existem poucos estudos a respeito da extração do óleo de moringa utilizando banho ultrassônico, esse trabalho tem como objetivo analisar os efeitos das variáveis, tempo, razão de mistura e temperatura na obtenção de óleo.

\section{METODOLOGIA}

\subsection{Matéria-prima}

Para a realização do estudo, as sementes de moringa da espécie Moringa oleifera Lamarck, utilizadas como matéria-prima para a extração do óleo, foram adquiridas na Fazenda Experimental de Iguatemi, pertencente à Universidade Estadual de Maringá - PR. No laboratório, passaram por um processo de limpeza para a remoção da casca, sendo armazenadas em refrigerador a $-15^{\circ} \mathrm{C}$.

\subsection{Processo de extração}

Antes de cada extração, as sementes foram trituradas e passadas por meio de uma peneira com Mesh 28, diâmetro de 0,59 mm, segundo Mani et al. (2007). Para a extração com ultrassom, foi utilizado um banho ultrassônico UltraCleaner modelo USC-1400 a, com uma frequência de $40 \mathrm{kHz}$, erlenmeyers de $125 \mathrm{~mL}$ com boca esmerilhada e cerca de $5 \mathrm{~g}$ de sementes. Para isto, fez-se o uso do solvente hexano Anidrol com 98,5\% de pureza.

Em seguida, cada amostra foi rotoevaporada para retirada do solvente, por cerca de 30 minutos a $70^{\circ} \mathrm{C}$. O cálculo do teor de óleo foi obtido pela equação abaixo:

$$
\% \text { Teor }=\frac{m_{\text {oleo }}}{m_{\text {semente }}} .100
$$

\subsection{Planejamento Experimental}

As condições experimentais foram otimizadas aplicando-se um planejamento estatístico de Delineamento Composto Central Rotacional (DCCR) $2^{3}$ fatorial aliado à Metodologia de Superfície de Resposta (MSR). O software Statistica 7.0 ® foi utilizado para realizar a análise de variância (ANOVA) e gerar superfícies de resposta. O modelo matemático definido foi considerado satisfatório quando os dados ANOVA revelaram um alto nível de significância estatística, com valores de $\mathrm{F}$ no nível de $95 \%$ de confiança e p-valores $<0,05$. As seguintes variáveis independentes foram analisadas: tempo de extração, razão de mistura (massa de amostra/volume de solvente) e temperatura do banho, conforme a Tabela 1. 
O teor de óleo obtido foi selecionado como reposta para combinação das variáveis independentes.

Para realização do delineamento utilizou-se 8 pontos fatoriais $(+1$ e -1$), 6$ pontos axiais $(+1,68$ e $-1,68)$ e 6 pontos centrais $(0)$, totalizando 20 experimentos. Cada ensaio foi feito em duplicata.

Tabela 1 - Níveis reais e codificados das variáveis independentes do planejamento experimental

\begin{tabular}{cllllc}
\hline & \multicolumn{5}{c}{ Níveis } \\
\hline Fator & $-1,68$ & -1 & 0 & 1 & 1,68 \\
Tempo $(\min )$ & 19,77 & 30 & 45 & 60 & 70,23 \\
Temperatura $\left({ }^{\circ} \mathrm{C}\right)$ & 18,18 & 25 & 35 & 45 & 51,82 \\
Razão de mistura $(\mathrm{g} / \mathrm{mL})$ & $1 / 4,6$ & $1 / 6$ & $1 / 8$ & $1 / 10$ & $1 / 11,4$ \\
\hline
\end{tabular}

\section{RESULTADOS E DISCUSSÕES}

O teor de óleo extraído variou de $25,75 \%$ a $39,80 \%$, para as combinações feitas entre temperatura do banho em ultrassom, tempo de extração e razão de mistura amostra/solvente. $\mathrm{O}$ maior valor de teor foi obtido no tempo de 60 minutos, temperatura de $25^{\circ} \mathrm{C}$ e razão de mistura 1:10. Mani et al. (1999) estudaram a extração do óleo de sementes de moringa por meio do aparelho Soxhlet, utilizando $250 \mathrm{ml}$ hexano e $20 \mathrm{~g}$ de sementes por um período de $4 \mathrm{~h}$, e relataram um teor de $33,10 \%$.

$\mathrm{Na}$ Tabela 2, estão apresentados os resultados da análise de variância (ANOVA) para a extração por ultrassom. Através da ANOVA, constatou-se que os parâmetros lineares razão de mistura e tempo são significativos, uma vez que o p-valor de é menor que 0,05 . Quanto às interações, apenas a de tempo e temperatura não é significativa.

Tabela 2 - Análise de variância para a extração por ultrassom utilizando sementes de moringa para a resposta teor de óleo (\%)

\begin{tabular}{cccccc}
\hline Fonte de variação & $\begin{array}{c}\text { Soma dos } \\
\text { quadrados }\end{array}$ & $\begin{array}{c}\text { Grau de } \\
\text { liberdade }\end{array}$ & $\begin{array}{c}\text { Soma dos } \\
\text { quadrados } \\
\text { médios }\end{array}$ & F & p-valor \\
\hline Tempo & 15,593 & 1 & 15,593 & 5,36284 & 0,031903 \\
Tempo $^{2}$ & 13,9153 & 1 & 13,9153 & 4,78583 & 0,041398 \\
Temperatura $^{\text {Temperatura }}$ & 9,9402 & 1 & 9,9402 & 3,41868 & 0,080085 \\
Razão & 9,637 & 1 & 9,637 & 3,3144 & 0,084464 \\
Razão $^{2}$ & 16,809 & 1 & 16,809 & 5,78105 & 0,026562 \\
Tempo*Temperatura & 1,331 & 1 & 1,331 & 0,45776 & 0,506827 \\
Tempo*Razão & 28,1064 & 1 & 2,2832 & 1,47311 & 0,239728 \\
Temperatura*Razão & 180,7016 & 1 & 180,7016 & 6,6665 & 0,005777 \\
\hline Falta de ajuste & 128,8754 & 5 & 25,7751 & 8,8647 & 0,000178 \\
Erro puro & 55,2445 & 19 & 2,9076 & & 0 \\
\hline
\end{tabular}


Nas Figuras 1 a 3 são apresentados os gráficos de superfícies de respostas, para analisar os efeitos das variáveis independentes sobre o teor de óleo extraído. Sendo que, para a obtenção das superfícies se fez necessário a escolha de dois fatores em estudo, ao passo que o terceiro foi mantido constante em seus níveis experimentais.

Figura 1 - Curva de superfície relacionando tempo e razão de mistura

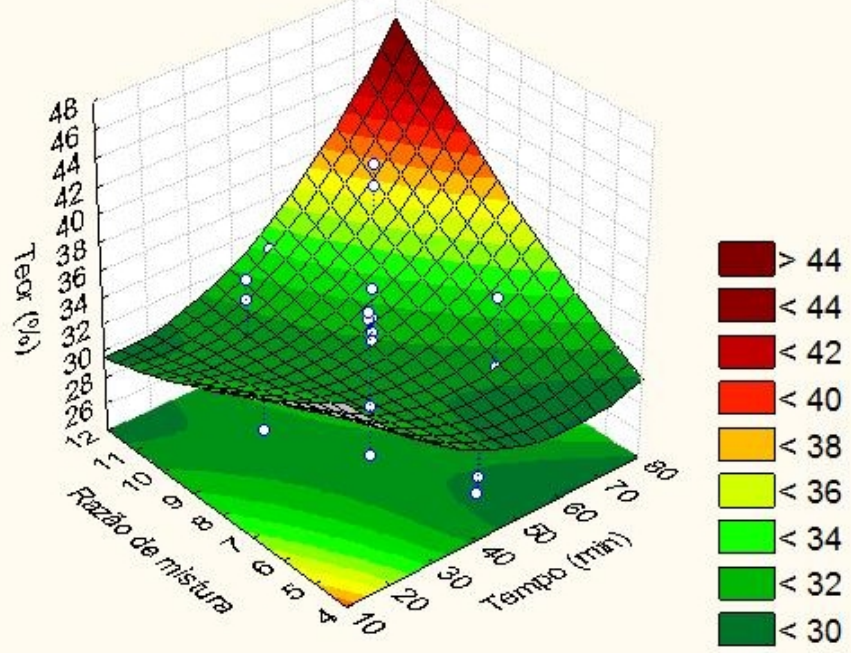

A Figura 1 mostra o efeito do tempo e razão de mistura no teor de óleo obtido, à temperatura de $35^{\circ} \mathrm{C}$. Foi observado que quando se aumenta ambas as variáveis, extraí-se uma maior quantidade de óleo. Tal que, o maior teor pode ser obtido em 80 minutos, a uma razão de mistura 1:12. Zhang et al. (2009) e Goula (2013), que trabalharam com amêndoa e semente de romã, respectivamente, atingiram resultados similares.

Figura 2 - Curva de superfície relacionando tempo e temperatura

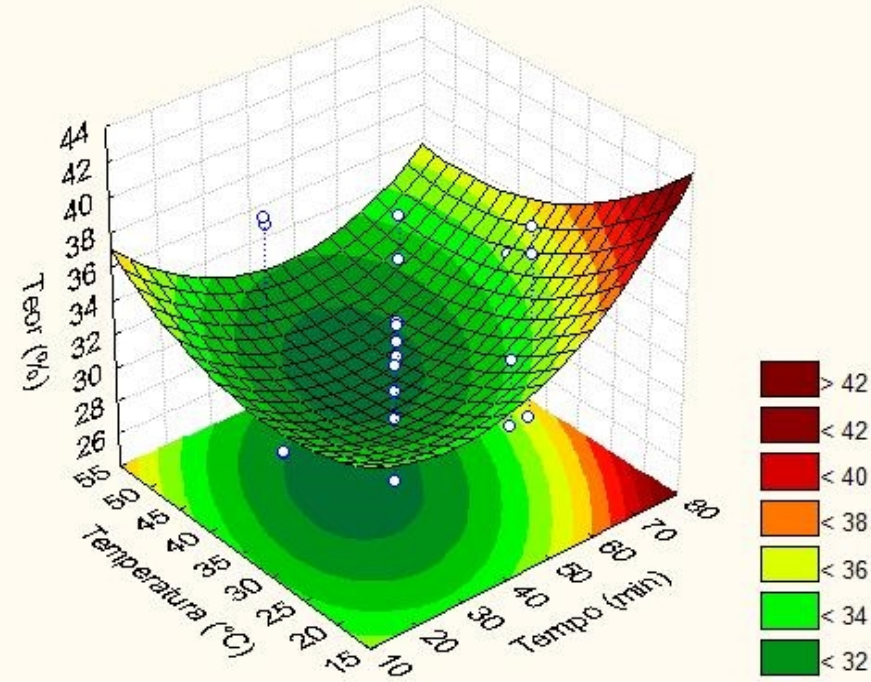


A Figura 2 relaciona o efeito do tempo e da temperatura no teor de óleo, com razão fixa 1:8. Nota-se que quanto maior o tempo e menor a temperatura, maior será a teor de óleo encontrado. De modo que, o maior teor será em 80 minutos, com temperatura $15^{\circ} \mathrm{C}$. Este resultado não é o esperado, já que o aumento da temperatura deve aumentar a solubilidade e o coeficiente de difusividade, entretanto Zhang et al. (2008) e Goula (2013), que estudaram linhaça e semente de romã, respectivamente, alcançaram resultados semelhantes a este e justificaram os valores, ao fato de que, a pressão de vapor do solvente aumentou com o aumento da temperatura e da pressão de vapor, influenciando a ocorrência e a intensidade da cavitação acústica.

Figura 3 - Curva de superfície relacionando temperatura e razão de mistura

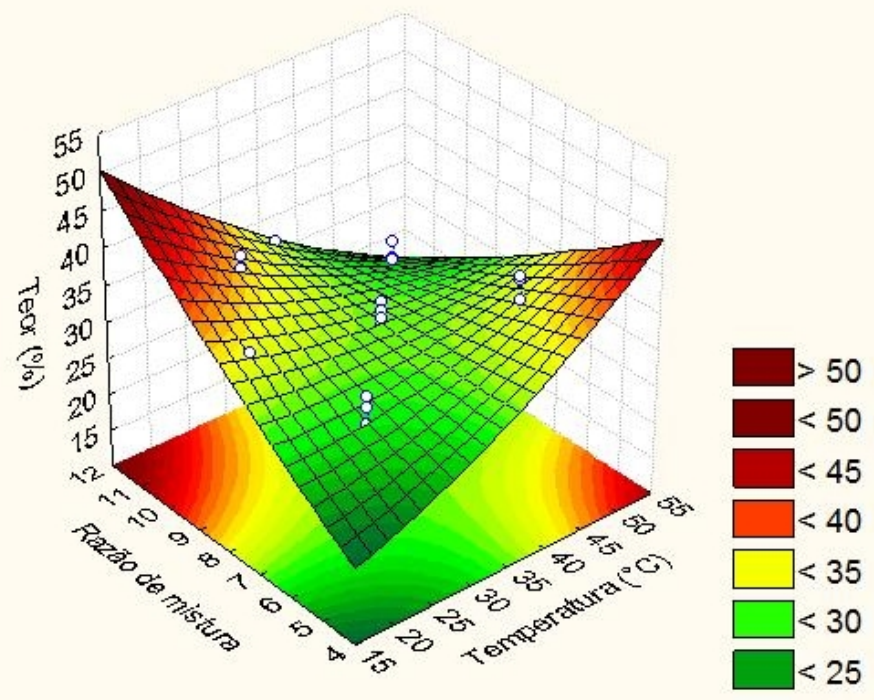

A Figura 3 apresenta a interação mais significativa, que relaciona a temperatura e a razão. Fixando o tempo em 45 minutos, pode-se constatar que, o aumento da razão de mistura e a baixa temperatura favorecem a obtenção de óleo.

\section{CONCLUSÃO}

Pelas análises estatísticas, por meio da metodologia de superfície de resposta, pode-se concluir que as melhores condições para a extração do óleo de moringa em ultrassom, usando o hexano como solvente, são: menor temperatura e maior razão de mistura e tempo. Deste modo, a obtenção de óleo por este método, quando comparado com métodos tradicionais de extração, é vantajoso, uma vez que se diminuiu a quantidade de solvente utilizado e, também, o tempo de extração.

\section{REFERÊNCIAS BIBLIOGRÁFICAS}

ANWAR, F.; BHANGER, M. I. Analytical Characterization of Moringa oleifera Seed Oil Grown in Temperate Regions of Pakistan. Journal of Agricultural and Food Chemistry, v.51, p.6558-6563, 2003. 
DAHOT, M.U. Vitamin contents of the flowers and seeds of Moringa oleifera L. Journal of Biochemistry, v.21, n.1-2, p.21-24, 1998.

GOULA, A. M. Ultrasound-assisted extraction of pomegranate seed oil - Kinetic modeling. Journal of Food Engineering, v.117, p.492-498, 2013.

MANI, S.; JAYA, S.; VADIVAMBAL, R. Optimization of solvent extraction of moringa (Moringa oleifera) seed kernel oil using response surface methodology. Food and Bioproducts Processing, v. 85, p.328-335, 2007.

MELECCHI, M. I. S. Caracterização química de extratos de Hibiscus tiliaceus L: estudo comparative de métodos de extração. Tese (Doutorado), Universidade Federal do Rio Grande do Sul, 2005.

OLIVEIRA, R. C. DE; BARROS, S. T. D. de; GIMENES, M. L. The extraction of passion fruit oil with green solvents. Journal of Food Engineering, v.117, p.458-463, 2013.

SANTANA, C. R.; PEREIRA, D. F.; ARAÚJO, N. A. de; CAVALCANTI, E. B.; SILVA, G. F. Caracterização físico-química da moringa. Revista Brasileira de Produtos Agroindustriais, v.12, n.1, p.55-60, 2010.

SARGENTI, S. R.; VICHNEWSI, W. Sonication and liquid chromatography as a rapid technique for extraction and fractionation of plant material. Phytochemical analysis, v.11, n.2, p.69-73, 2000.

TIAN, Y.; XU, Z.; ZHENG, B.; LO, Y. M., Optimization of ultrasonic-assisted extraction of pomegranate (Punica granatum L.) seed oil. Ultrasonics Sonochemistry, v.20, p.202208, 2013.

ZHANG, Q.; ZHANG, Z.; YUE, X.; FAN, X.; LI, T.; CHEN, S., Response surface optimization of ultrasound-assisted oil extraction from autoclaved almond powder. Food Chemistry, v.116, p.513-518, 2009.

ZHANG, Z.; WANG, L.; LI, D.; JIAO, S. S.; CHEN, X. D.; MAO, Z., Ultrasound-assisted extraction of oil from flaxseed. Separation and Purification Technology, v.62, p.192$198,2008$. 